Classification

Physics Abstracts

8.824

\title{
VIBRATIONS DE RÉSEAU DANS LE FLUOROBENZÈNE
}

\author{
M. PIERRE \\ Laboratoire de Spectrométrie Physique (*) \\ Université Scientifique et Médicale de Grenoble \\ B.P. 53, 38041 Grenoble Cedex, France \\ (Reçu le 28 juin 1976, accepté le 9 septemhre 1976)
}

\begin{abstract}
Résumé. - Une analyse des vibrations principales de réseau du fluorobenzène est proposée, utilisant les données des spectres Raman du polycristal à température variable entre $15 \mathrm{~K}$ et $150 \mathrm{~K}$; un calcul de modes normaux est effectué à partir des paramètres d'agitation thermique.

Abstract. - An interpretation of the fundamental lattice vibrations of solid fluorobenzene is deduced from an experimental investigation of Raman polycrystal line spectra at different temperatures between $15 \mathrm{~K}$ and $150 \mathrm{~K}$. A quantitative study of normal modes is presented involving mean square amplitudes of an oscillating rigid molecule.
\end{abstract}

1. Introduction. - Lors d'une étude antérieure [1] $\mathrm{du}$ spectre d'absorption du monocristal de fluorobenzène dans le proche ultraviolet, nous avons interprété la transition électronique pure $\pi \rightarrow \pi^{*}$ ${ }^{1} A_{1} \rightarrow{ }^{1} B_{1}$ en faisant intervenir la localisation de l'exciton. Nous avons montré comment les vibrations de réseau qui limitent l'extension de la zone d'excitation contribuent à l'élargissement des bandes spectrales lorsque la température augmente.

Il est nécessaire, pour arriver à une meilleure compréhension de ce mécanisme, d'avoir une bonne connaissance des modes de vibration intermoléculaires. Il n'existe jusqu'à présent pour ceux-ci que des données fragmentaires $[2,3,4]$. Nous avons entrepris une étude, à différentes températures, des spectres de diffusion Raman du fluorobenzène en phase polycristalline, afin d'identifier les vibrations principales intermoléculaires et suivre leur comportement en fonction de la température.

La figure 1 reproduit les spectres Raman de diffusion (nos mesures [5]) pour le polycristal à $15 \mathrm{~K}$ et pour le liquide à température ambiante; la figure 2 représente le spectre Raman de basse fréquence obtenu pour le polycristal à $99 \mathrm{~K}$ [5] et le compare au spectre d'absorption observé à $100 \mathrm{~K}$ dans l'infrarouge lointain [2].

Les vibrations intramoléculaires, dont la fréquence est supérieure à $200 \mathrm{~cm}^{-1}$, conservent dans le cristal pratiquement la même fréquence et la même activité que dans le liquide, la valeur de l'éclatement d'échange

(*) Associé au C.N.R.S.

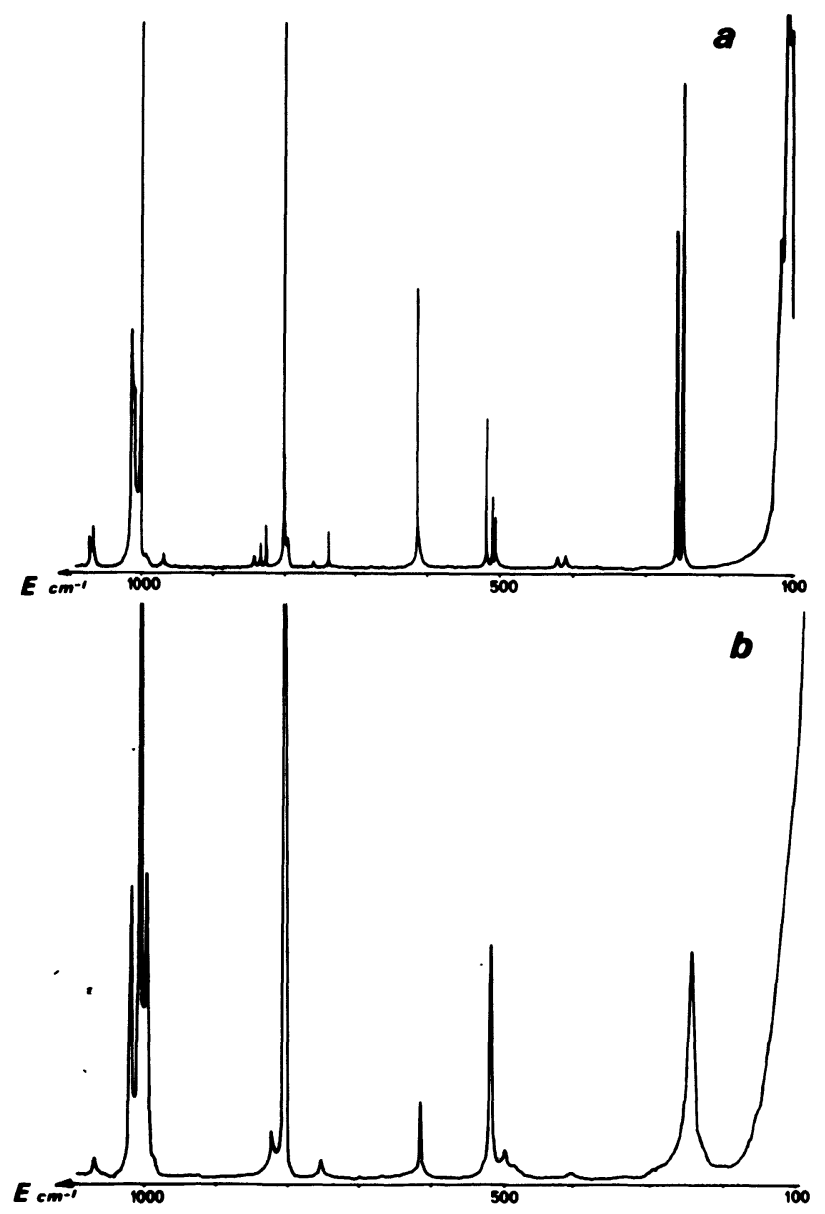

FIG. 1. - Comparaison des spectres de diffusion Raman du fluorobenzène polycristallin $(15 \mathrm{~K})(a)$; du liquide à température ambiante [5] (b). La raie excitatrice est choisie comme origine des fréquences. 


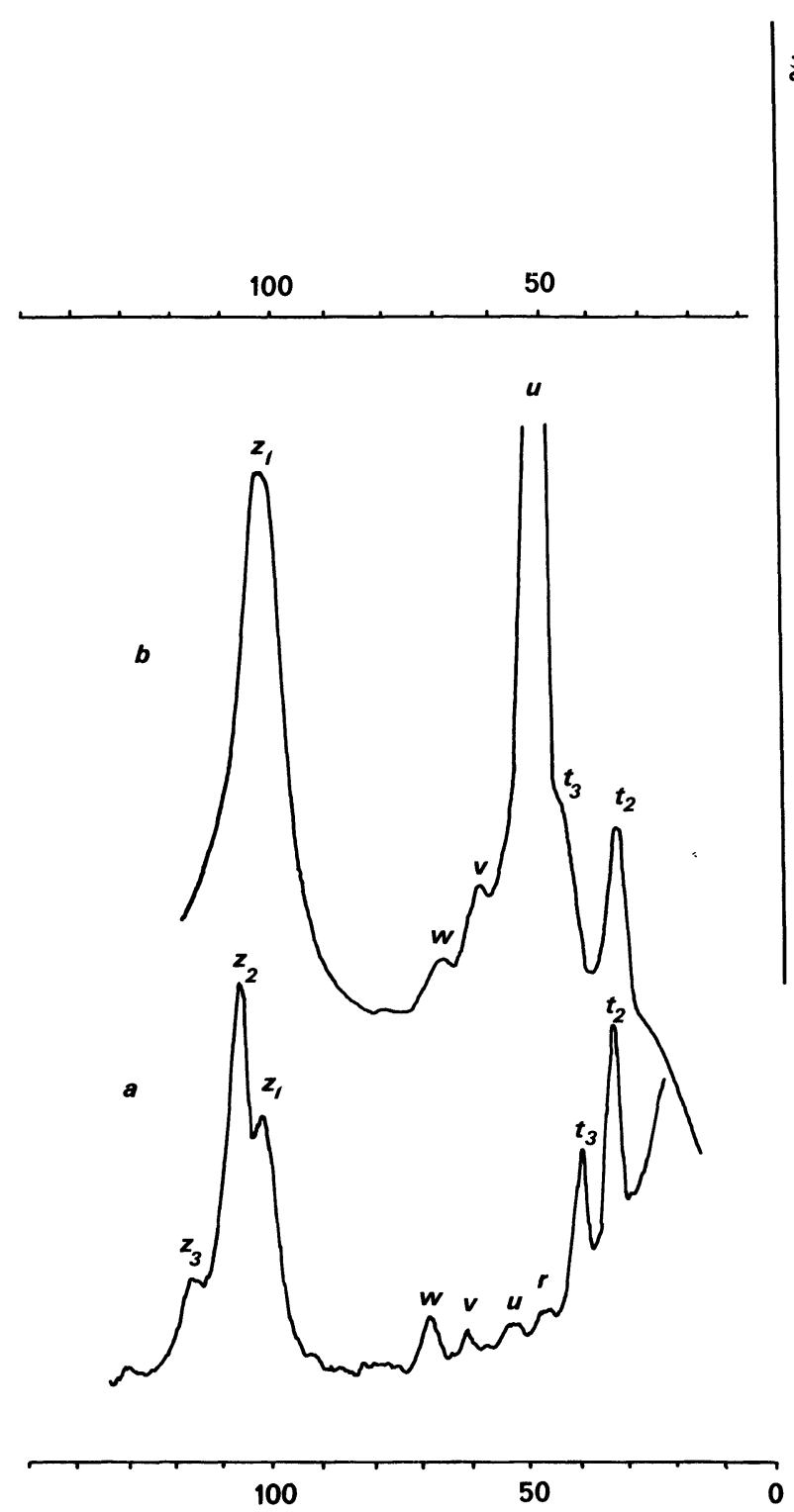

FIG. 2. - Comparaison des spectres Raman de basse fréquence [5] $(99 \mathrm{~K})(a)$ et infrarouge lointain [2] $(100 \mathrm{~K})(b)$ pour le monofluorobenzène polycristallin.

excédant rarement $5 \mathrm{~cm}^{-1}$ (Tableau I et Fig. 1). Ces résultats mettent en évidence la faible influence du champ cristallin, même sur les vibrations intramoléculaires de basse fréquence.
2. Calcul des fréquences de réseau. - $\mathrm{La}$ structure $\$$ cristalline du monofluorobenzène est maintenant bien connue [6,7]. Le cristal appartient à l'hémiédrie tétragonale correspondant au groupe spatial $\mathrm{D}_{4}^{4}$ ou $\mathrm{D}_{4}^{8}\left(\begin{array}{llllllll}\mathrm{P} & 4_{1} & 2 & 2 & \text { ou } \mathrm{P} & 4_{3} & 2 & 2\end{array}\right)$ avec 4 molécules par maille (Fig. 3). La symétrie du site est $\mathrm{C}_{2}$ : la molécule engagée dans le cristal subit une faible déformation par rapport à la phase vapeur mais conserve son axe de symétrie $z$ (Fig. 4).
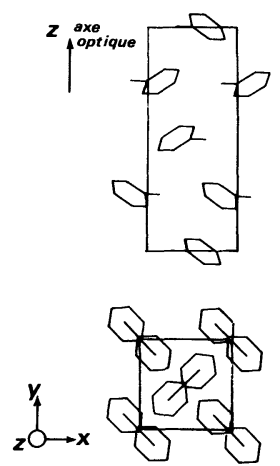

FIG. 3. - Disposition des quatre molécules de monofluorobenzène dans la maille quadratique.

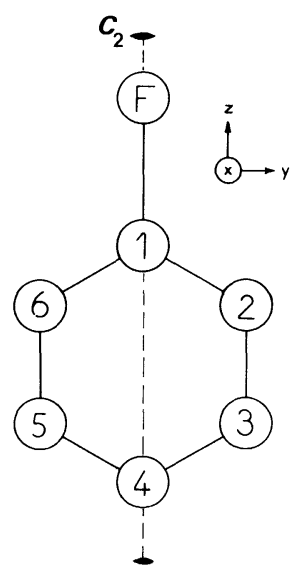

FIG. 4. - Molécule de fluorobenzène : l'axe $C_{2}$ est confondu avec $z$.

Nous avons cherché à évaluer par le calcul les fréquences et les vecteurs propres d'oscillation du réseau, à partir des valeurs obtenues en diffraction de rayons $X$ pour les paramètres d'agitation thermique [7]. Dans les conditions de l'expérience

\section{TABLEAU I}

Comparaison entre les fréquences de vibration du liquide (à la température ambiante) et du cristal (à $15 \mathrm{~K})$ pour le monofluorobenzène

Vibration

Fréquence en $\mathrm{cm}^{-1}$ dans le liquide

Fréquence moyenne en $\mathrm{cm}^{-1}$ dans le cristal $T=15 \mathrm{~K}$

Eclatement maximum entre composantes en $\mathrm{cm}^{-1}$

$\begin{array}{clllllllccc}16 \mathrm{~b} & 15 & 10 \mathrm{~b} & 6 \mathrm{a} & 6 \mathrm{~b} & 11 & 12 & 10 \mathrm{a} & 1 & 18 \mathrm{a} & 18 \mathrm{~b} \\ 242 & 407 & 499 & 519 & 613 & 754 & 806 & 829 & 1009 & 1021 & 1065 \\ 253 & 409 & 506 & 514 & 612 & 739 & 803 & 833 & 1000 & 1017 & 1069\end{array}$

$\begin{array}{lllllllllll}6 & 0 & 4 & 0 & 0 & 0 & 0 & 8 & 0 & 0 & 4\end{array}$ 
( $T=168 \mathrm{~K}$, grande séparation énergétique entre vibrations moléculaires et vibrations de réseau), les oscillations atomiques résultent en majeure partie des fluctuations de position de la molécule considérée comme un bloc rigide [8, 9], que l'on représente habituellement par les trois grandeurs tensorielles :

- $T$ exprimant la fluctuation translationnelle,

- $L$ la fluctuation librationnelle,

- $S$ la corrélation entre fluctuations de translation et de pivotement [8].

Les modes et les fréquences propres d'oscillation s'obtiennent en général par diagonalisation séparée des matrices $T$ et $L$. Nous avons constaté que l'accord avec les fréquences expérimentales est meilleur si l'on tient compte des corrélations entre mouvements de translation et de pivotement, en diagonalisant directement la matrice obtenue à partir de l'analyse des paramètres d'agitation thermique des atomes :

$$
V^{2}=\left(\begin{array}{ll}
L & S \\
\tilde{S} & T
\end{array}\right)\left({ }^{1}\right) .
$$

( $\left.{ }^{1}\right)$ Dans ce calcul, nous n'avons pas distingué les différences de peuplement thermique entre les différentes oscillations. Le paramètre arbitraire $\operatorname{Tr} S$ a été choisi égal à 0 .
Le tableau II compare les résultats obtenus par les deux méthodes de calcul. La description des modes d'oscillation qu'il fournit en termes de vecteurs propres, montre que les trois fréquences les plus élevées correspondent à des pivotements moléculaires et les trois basses fréquences à des oscillations de translation.

3. Analyse des spectres de basse fréquence. Conformément à la corrélation (Tableau III) entre le groupe moléculaire $C_{2 v}$ et le groupe facteur du cristal $\mathrm{D}_{4}$ par l'intermédiaire du site (Fig. 4), on constate que les 6 oscillations moléculaires donnent naissance à 16 fréquences optiques dont 13 sont actives en diffusion Raman et 8 en absorption infrarouge [10].

Il est intéressant de noter que les 5 vibrations qui apparaissent simultanément en absorption infrarouge et en diffusion Raman correspondent à la représentation $\mathrm{E}$ doublement dégénérée du groupe facteur. Elles couplent la translation $T_{z}$ ou la rotation $R_{z}$ aux autres translations et librations.

Le tableau IV regroupe les résultats obtenus en Raman à $15 \mathrm{~K}$ (nos mesures), en infrarouge à $100 \mathrm{~K}$ [2] et en absorption ultraviolette à $4 \mathrm{~K}$ [1]. Sur les 16 pho-

\section{TABleaU II}

Comparaison des deux calculs (avec et sans couplage translation-rotation) exprimant les fluctuations de position de la molécule de fluorobenzène dans le cristal à $168 \mathrm{~K}$. Pour chaque méthode de calcul, on indique successivement la fréquence propre d'oscillation en $\mathrm{cm}^{-1}$ et les différentes composantes rotationnelles et translationnelles du vecteur propre correspondant, exprimées par rapport aux axes d'inertie de la molécule au repos.

Calcul sans couplage rotation-translation Fréquences

\begin{tabular}{ccccccc} 
propres en cm $\mathrm{cm}^{-1}$ & $\mathrm{R}_{\boldsymbol{x}}$ & $\mathrm{R}_{\boldsymbol{y}}$ & $\mathrm{R}_{z}$ & $\mathrm{~T}_{x}$ & $\mathrm{~T}_{y}$ & $\mathrm{~T}_{z}$ \\
\hline- & - & - & - & - & - & - \\
36 & & & & 0,0 & 0,0 & 1,0 \\
37 & & & & 0,12 & 0,99 & 0,0 \\
46 & & & & 0,99 & 0,12 & 0,0 \\
63 & 0,99 & 0,13 & 0,0 & & & \\
64 & 0,13 & 0,99 & 0,0 & & & \\
85 & 0,0 & 0,0 & 1,0 & & &
\end{tabular}

Calcul avec couplage translation-rotation

Fréquences

propres en $\mathrm{cm}^{-1}$
-
17
24
35
60
71
117

TABLEAU III

Table de corrélation entre le groupe moléculaire et le groupe facteur du fluorobenzène mettant en évidence la classification des mouvements externes

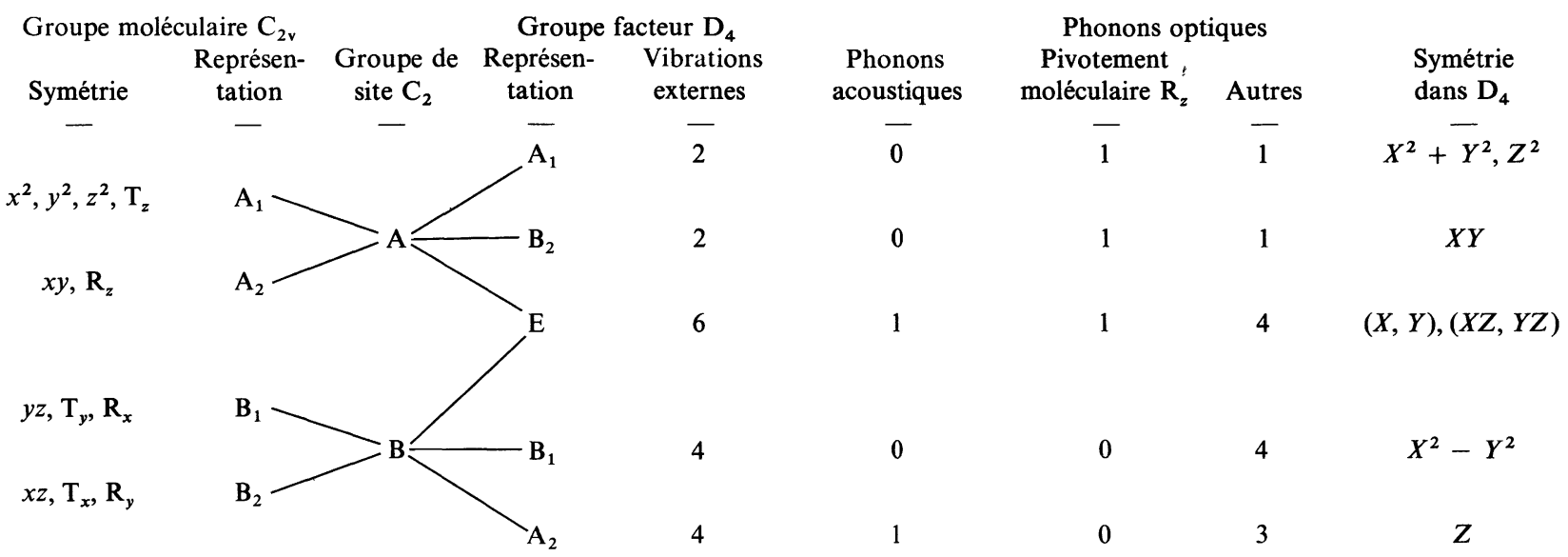




\section{TABLEAU IV}

Attribution proposée pour les principales vibrations de réseau du monofluorobenzène.

$$
\mathrm{I} \text { : intense ; } \mathrm{m} \text { : intensité moyenne ; } \mathrm{f} \text { : faible intensité }
$$

$\left.\begin{array}{cccc} & \text { Raman 15 K } & \text { Infrarouge } 100 \mathrm{~K} & \text { Ultraviolet } 4 \mathrm{~K} \\ \mathrm{t}_{1} & - & - & - \\ \mathrm{t}_{2} & - & - & 16 \mathrm{I} \\ \mathrm{t}_{3} & 35 \mathrm{I} & 37 \mathrm{I} & 31 \mathrm{f} \\ \mathrm{r} & 42 \mathrm{I} & 46 \mathrm{~m}\left(^{*}\right) & 43 \mathrm{~m}\end{array}\right\}$

Prédominance des oscillations moléculaires de translation

Composantes des pivotements autour d'axes perpendiculaires à $z$ couplés avec des translations

Composantes du pivotement autour de l'axe moléculaire $z$ nons attendus, 6 n'apparaissent pas. L'analyse que nous proposons diffère sensiblement de celles qui ont été présentées antérieurement par Fleming et al. [2] et par Nevzorov et al. [3], basées sur des observations faites uniquement en infrarouge [2] ou en Raman [3].

La comparaison des résultats expérimentaux et des données du calcul (Tableau II) permet de faire correspondre sans ambiguité les fréquences observées aux mouvements moléculaires de translation et de pivotement (Tableau IV). Mais l'attribution précise de ces fréquences aux différents modes propres phononiques du cristal est pratiquement impossible en l'absence d'observations en lumière polarisée.

3.1 Translations T. - La plus basse fréquence calculée (Tableau II) qui correspond à une translation perpendiculaire à $z$ (Fig. 4) n'est pas observable dans les spectres Raman et infrarouge, mais elle donne naissance à une bande intense en absorption ultraviolette.

Les deux raies fines $\left(t_{2}\right)$ et $\left(t_{3}\right)$ observées en Raman et infrarouge au voisinage de $35-40 \mathrm{~cm}^{-1}$ correspondent certainement aux deux oscillations de translation, de fréquences calculées 24 et $35 \mathrm{~cm}^{-1}$ (Tableau II). L'activité simultanée en infrarouge et Raman laisse à penser que les fréquences observées sont à attribuer aux modes doublement dégénérés $\mathrm{E}$; on ne peut toutefois exclure la possibilité d'une attribution à des modes phononiques de symétrie différente en Raman et infrarouge, dont les fréquences seraient exceptionnellement voisines.

3.2 Pivotements $\mathrm{R}_{x}$ ET $\mathrm{R}_{y}$. - Ils sont observés entre 50 et $75 \mathrm{~cm}^{-1}$ (bandes $\mathrm{u}, \mathrm{v}, \mathrm{w}, \mathrm{r}$ ). La bande (u) très intense en infrarouge, mais faible en Raman, correspond vraisemblablement à l'excitation d'un mode phononique de symétrie $A_{2}$.

On constate que les librations $\mathbf{R}_{x}, \mathbf{R}_{\boldsymbol{y}}$ paraissent avoir une faible influence sur la polarisabilité de la molécule, puisqu'elles sont moins actives en Raman que les autres mouvements moléculaires. Par contre, l'une d'elles entraîne une variation importante du moment dipolaire : il pourrait s'agir du pivotement $\mathbf{R}_{\boldsymbol{x}}$ qui provoque une rotation gênée de la molécule dans son plan, et dont la fréquence calculée est en bon accord avec la valeur expérimentale.

3.3 Pivotement AUtouR DE $z$. - Cette libration donne naissance à trois composantes en Raman (Fig. 2); une seule d'entre elles $\left(z_{1}\right)$, attribuée par conséquent à un mode de symétrie $\mathrm{E}$, apparaît en infrarouge. L'identification des deux autres composantes $\left(z_{2}, z_{3}\right)$ est justifiée par un calcul de dédoublement excitonique dans le modèle dipolaire [1]; en supposant le moment dipolaire dérivé dirigé le long de $z$, on trouve en effet que les trois modes se situent dans l'ordre suivant des fréquences croissantes : E doublement dégénéré, $A_{1}$ totalement symétrique, $B_{2}$ non symétrique, le mode $A_{1}$ étant plus voisin en fréquence de $\mathrm{E}$ que de $\mathrm{A}_{2}$.

Si l'on se réfère aux observations faites sur les autres dérivés benzéniques monosubstitués ou paradisubstitués, l'activité en infrarouge du pivotement $\mathbf{R}_{\mathbf{z}}$ est exceptionnellement grande pour le fluorobenzène. Ce fait traduit vraisemblablement l'existence d'un couplage vibrationnel : celui qui a lieu avec les autres vibrations de réseau est négligeable (Tableau II); par contre un couplage avec des vibrations moléculaires propres totalement symétriques, est rendu possible par l'identité entre l'axe de libration et l'axe $\mathrm{C}_{2}$ de la molécule.

On sait que pour les molécules dont un axe de libration se confond pratiquement avec un axe d'inertie, il apparaît souvent, même à basse température, un phénomène de réorientation autour de cet axe $[11,12,13,14]$. Pour le fluorobenzène, une réorientation autour de l'axe $z$ jouerait notablement sur la structure excitonique de la transition électronique ${ }^{1} \mathrm{~A}_{1} \rightarrow{ }^{1} \mathrm{~B}_{1}$ dont le moment est perpendiculaire à $z$. 
4. Influence de la température. - La figure 5 reproduit les enregistrements des spectres Raman obtenus à différentes températures entre 15 et $150 \mathrm{~K}$ : on remarque une sensibilité particulière du massif $\left(z_{1}\right.$, $\left.z_{2}, z_{3}\right)$ à la variation de $T$. Ce comportement privilégié
150 100 50

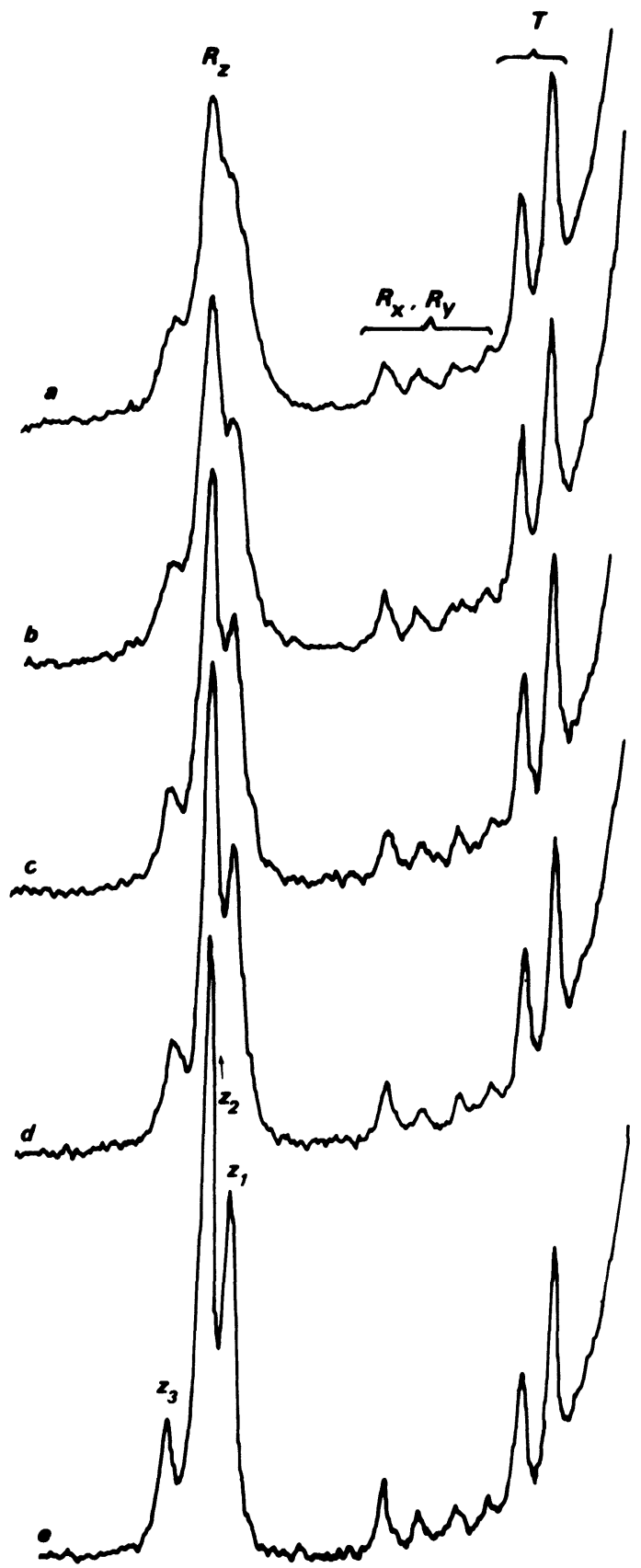

\section{0}

100 des modes associés au pivotement $R_{z}$ est confirmé par l'évolution, en fonction de la température, de la hauteur de pic et de la largeur de bande (Fig. 6 et $7 a$ ). L'intensité intégrée pour le massif $R_{z}$ reste pratiquement constante dans l'intervalle de température considéré, mais la répartition spectrale d'intensité se modifie notablement. De 15 à $80 \mathrm{~K}$ environ, la hauteur des pics $\left(z_{1}, z_{2}, z_{3}\right)$ subit une diminution rapide, comparée à celle que nous observons pour les autres modes (Fig. 6); elle décroît ensuite plus lentement et se stabilise à des températures supérieures à $150 \mathrm{~K}$ [3] $\left(^{2}\right)$. De façon parallèle, la largeur de bande évaluée pour $\left(z_{2}\right)$ présente aux alentours de $80 \mathrm{~K}$ une rupture dans sa variation croissante en fonction de $T$ (Fig. 7a).

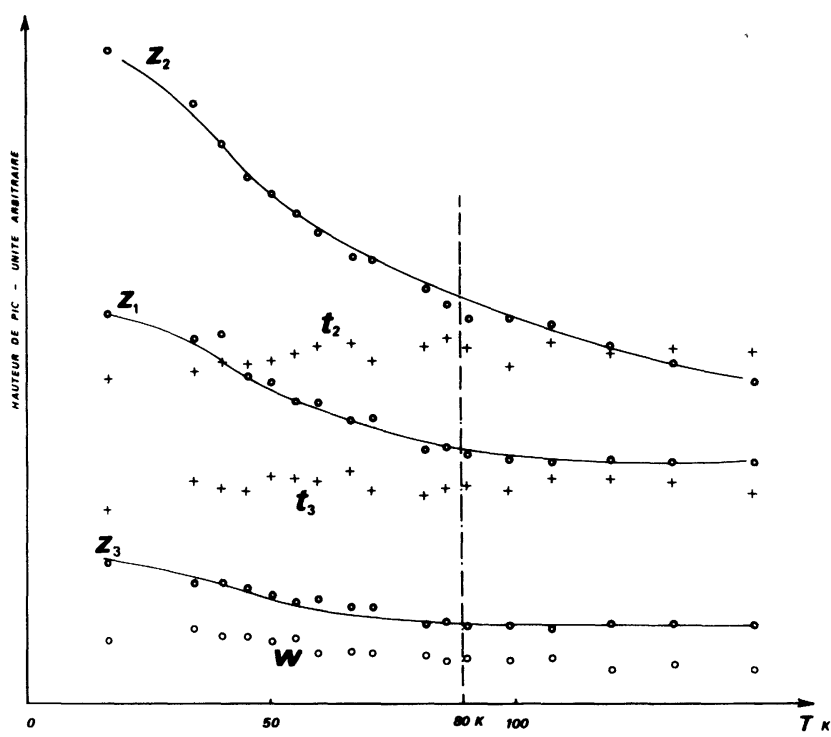

Fig. 6. - Variation de la hauteur du pic (unités arbitraires) en fonction de la température pour différentes raies du spectre Raman.

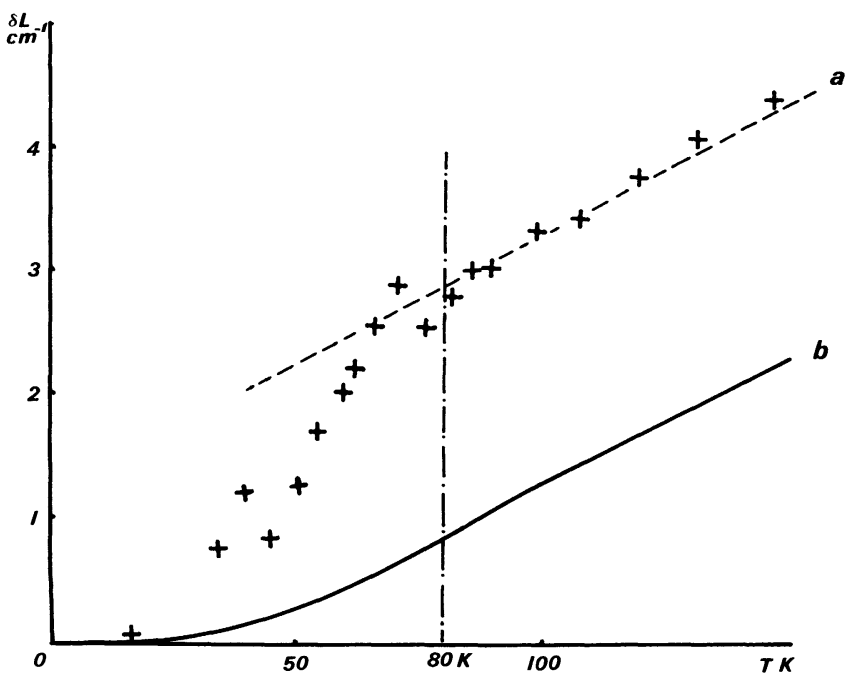

Fig. 7. - Augmentation de la largeur de raie du mode $z_{2}$ avec la température : $a$ ) courbe expérimentale ; $b$ ) courbe théorique (oscillateur anharmonique). exprimée en $\mathrm{cm}^{-1}$ a A) a) $148 \mathrm{~K}$; b) $107 \mathrm{~K}$; c) $67 \mathrm{~K}$; d) $45 \mathrm{~K}$;e) $15 \mathrm{~K}$. Les mesures ont été effectuées sur un double monochromateur Coderg PH.1, avec un laser Spectra Physics 16 de $500 \mathrm{~mW}$. $\left.{ }^{2}\right)$ La température de fusion du fluorobenzène est $231 \mathrm{~K}$. 
Il nous a paru intéressant de rapprocher ces observations de celles faites par d'autres auteurs - voir en particulier $[10,11]$ - qui ont remarqué que :

a) En l'absence de réorientations moléculaires, les modifications avec la température du spectre de diffusion Raman sont essentiellement dues à l'anharmonicité des vibrations de réseau. Lorsque la température augmente il se produit : - une faible diminution de la fréquence, en relation avec une faible variation des paramètres de la maille ; - un élargissement des raies de diffusion proportionnel à la température.

b) Une réorientation des molécules (sans changement de phase cristallographique) ne semble pas avoir d'influence déterminante sur la variation de la fréquence de libration autour de l'axe concerné; elle entraîne cependant une fluctuation de la fréquence qui se traduit par une augmentation de la largeur de raie de diffusion. Dans le modèle du rotateur de Frenkel $[15,16]$, cette fluctuation est proportionnelle à $\exp [-v / T]$ où $v$ est la hauteur de la barrière de potentiel s'opposant au retournement de la molécule, $T$ la température.

Nous observons effectivement pour les fréquences des modes associés au pivotement $R_{z}$, une diminution linéaire en fonction de la température (Fig. 8); bien

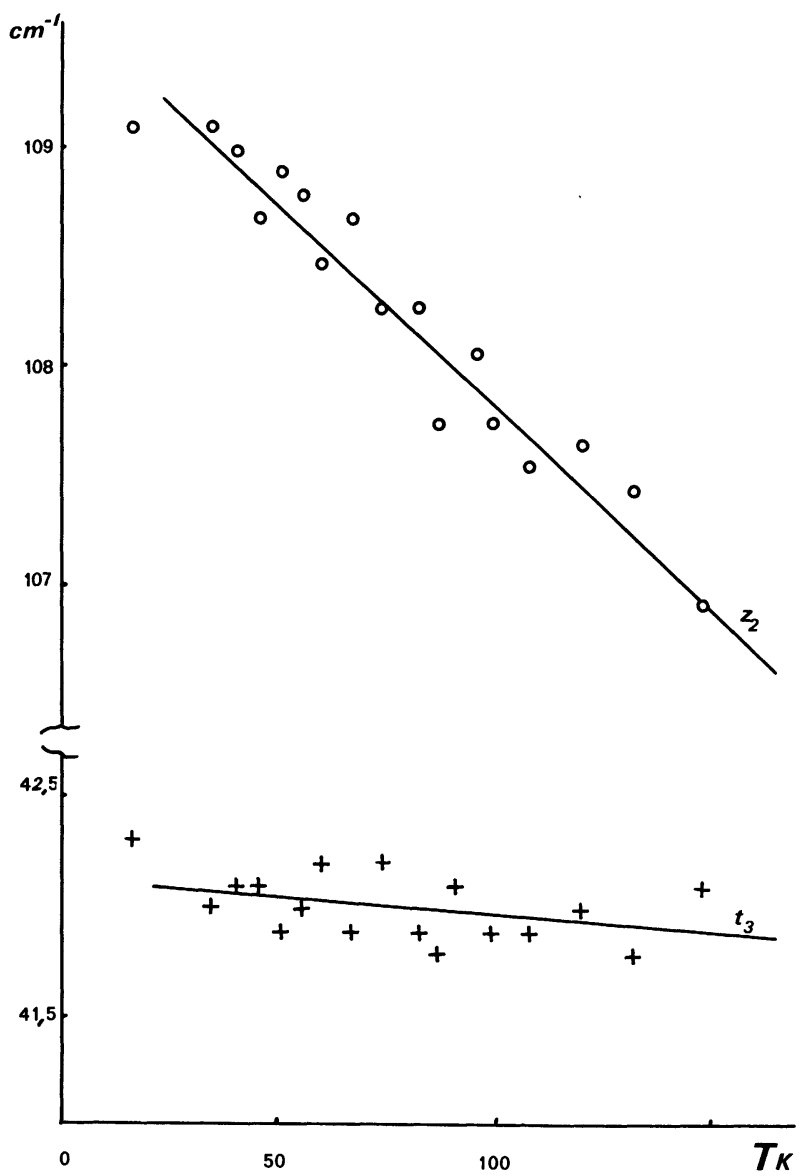

FIG. 8. - Variation de la fréquence $\left(\mathrm{en}^{-1}\right.$ ) en fonction de la température pour les modes $t_{3}$ et $z_{2}$. que cette diminution soit plus importante que celle relevée pour les autres modes, sa variation relative $\left(1,5 \times 10^{-4}\right)$ est du même ordre que le coefficient de dilatation volumique (environ $10^{-4}$ ) des cristaux moléculaires.

Pour les hautes températures (supérieures à $80 \mathrm{~K}$ ), nous avons cherché à rendre compte de la variation linéaire de la largeur des bandes à partir d'un modèle anharmonique. Le potentiel est supposé indépendant de la température, le peuplement des différents niveaux vibrationnels obéit à la statistique de BoseEinstein, pour une forme de raie à $0 \mathrm{~K}$ lorentzienne symétrique (Fig. 9). Le coefficient d'anharmonicité a été évalué de manière à obtenir le meilleur accord avec l'expérience; sa valeur $(0,025)$ est voisine de celle que l'on peut trouver dans la littérature pour d'autres cristaux moléculaires. La courbe $7 b$ reproduit la largeur de bande calculée.
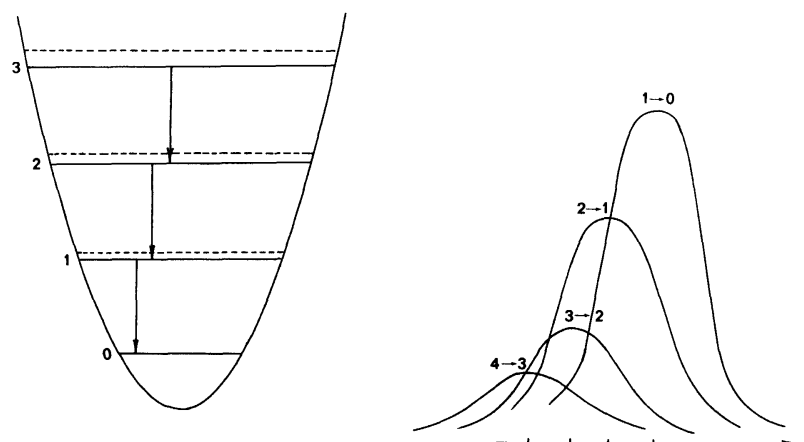

Fig. 9. - Construction du modèle anharmonique par superposition de raies de forme lorentzienne.

Pour des températures inférieures à $80 \mathrm{~K}$, il est possible que la variation de largeur de bande reflète un phénomène de réorientation des molécules autour de leur axe $\mathrm{C}_{2}(z)$; nous avons déjà indiqué l'éventualité de tels retournements moléculaires. Toutefois, si l'on applique au fluorobenzène le modèle du rotateur de Frenkel, on obtient pour la barrière de potentiel $v$ une valeur d'environ $110 \mathrm{cal} / \mathrm{mole}$, nettement trop faible par rapport à celles données généralement dans la littérature (quelques kilocalories par mole) pour des composés de structure moléculaire voisine [14]. Il convient de remarquer que l'on entend habituellement par phénomène de réorientation de la molécule un véritable changement de position dans l'espace du squelette nucléaire. Nous pensons que dans le

cas étudié la réorientation observée traduit un changement de signe de la fonction d'onde considérée, induit par un mécanisme excitonique. Effectivement, dans le cas d'un retournement réel de la molécule, le modèle de Pauling pour la rotation gênée $[17,18]$ avec un potentiel de la forme $\frac{1}{2} v(1-\cos 2 \theta)$ conduit à une valeur élevée de la barrière

$$
v \simeq 29 \mathrm{kcal} / \mathrm{mole},
$$

lorsqu'il est appliqué à la fréquence de $109 \mathrm{~cm}^{-1}$ correspondant au pivotement $\mathbf{R}_{\mathbf{z}}$. 
Il est très remarquable que cette même température de $80 \mathrm{~K}$ se retrouve dans l'étude de la structure excitonique de la bande de transition électronique pure du spectre d'absorption ${ }^{1} \mathrm{~A}_{1} \rightarrow{ }^{1} \mathrm{~B}_{1}$; dans le domaine de températures inférieur à $80 \mathrm{~K}$, on observe en effet une modification de la structure excitonique avec, en particulier, un élargissement rapide des bandes quand la température augmente [1]. Ce comportement n'est pas en contradiction avec le rôle des réorientations moléculaires, prépondérant en dessous de $80 \mathrm{~K}$. Les retournements de moment dipolaire que celles-ci entraînent conduisent à une modification notable des mécanismes d'échange et, lorsque le temps de transfert de l'excitation électronique d'une molécule à l'autre devient inférieur à l'intervalle de temps entre deux réorientations moléculaires, il y a localisation de l'exciton, d'où élargissement des bandes. Une analyse détaillée de la structure excitonique est en cours, afin de mieux préciser ce mécanisme.

Remerciements. - L'auteur remercie vivement Madame J. Kahane pour l'aide apportée dans l'élaboration de ce travail.

\section{Bibliographie}

[1] Pierre, M., J. Physique 36 (1975) 1095.

[2] Fleming, J. W., Turner, P. A., Chantry, G. W., Mol. Phys. 19 (1970) 853.

[3] Nevzorov, B. P., Sechkarev, A. V., Sov. Phys. J. 14 (1973) 199.

[4] Dumas, G. G., Marcou, A., Chedin, M. P., Freymann, R., C.R. Hebd. Séan. Acad. Sci. B 267 (1968) 267.

[5] PierRe, M., XIth European Congress on Molecular Spectroscopy, Tallinn (1973).

[6] Henshaw, D. E., Acta Crystallogr. 14 (1961) 1080.

[7] Clavaguera Plaja, N., Thèse, Orsay (1970).

[8] Schomaker, V., Trueblood, K. N., Acta Crystallogr. B 24 (1968) 63.

[9] Sheringer, C., Acta Crystallogr. A 29 (1973) 554.
[10] Poulet, H., Mathieu, J. P., Spectres de vibration et symétrie des cristaux (Gordon \& Breach, Paris) 1970.

[11] Korshunov, A. V., Bondarev, A. F., Opt. Spectrosc. 15 (1963) 98.

[12] Wang, C. H., Fleury, P. A., Light scattering spectra of solids (New York) 1969.

[13] Colombo, L., Moreau, D., C. R. Hebd. Séan. Acad. Sci. 265 (1967) 487.

[14] Bazhulin, P. A., Rakhimov, A. A., Sov. Phys. Solid State 7 (1966) 1681.

[15] Fourme, R., Thèse, Paris (1970).

[16] Rakov, A. V., Opt. Spectrosc. 10 (1961) 377.

[17] Pauling, L., Phys. Rev. 36 (1930) 430.

[18] Herschbach, D. R., J. Chem. Phys. 27 (1957) 975. 